\section{A bacterial way of life}

SiR-A coherent concept of the bacterial world is long overdue. I feel this is largely because of the tendency to investigate bacterial strains out of the context in which they occur in nature. If, however, they are considered as adaptable chimaeras, a coherent concept emerges.

Bacteria live mostly in mixed local communities made up of metabolically different strains which support one another ${ }^{1}$. Each community functions as a single organism, with an extremely efficient division of labour. Such integrated entities are found, for example, in soil and digestive tracts, and represent a bacterial solution to the need for a complex life strategy involving pluricellular participation. It is the equivalent of an individual plant or animal ${ }^{2}$. New bacterial communities may be rapidly improvised by local selective pressures. They continually correct the mixture of their constituents by replacing less efficient strains with more appropriate ones. Therefore these dynamic communities - consisting of more than one genetic type of cells and able to switch their ecto-symbiotic strains - are adaptable chimaeras.

Similarly, at the cellular level, each bacterium is enriched by the temporary presence of one or more visiting genes, mostly small replicons - circular, selfreplicating DNA molecules (prophages or plasmids). Their 'converting' genes encode potentially useful attributes, accessory to essential ones encoded by the large replicon ('chromosome' or nucleoid) of the cell. As soon as its usefulness disappears, a small replicon can be eliminated by 'curing', often to be replaced by a more appropriate one from the large pool that exists in many other strains. Specific receptors for such visiting genes are present at the surface of any bacterial cell. All categories of bacteria, including Archaebacteria $^{3.4}$, possess at least one plasmid. Moreover, at least one in three strains contains one or more prophages (polylysogeny). Non-self-replicating DNA fragments may also be exchanged between different bacterial strains, although less frequently. When a favourable gene arrives in a cell from another strain, the newly formed cellular entity - also a temporary, adaptable chimaera - rapidly multiplies. When threatened by antibacterial substances, such as drugs ${ }^{2}$ and active biological products used in agriculture, industrial or natural waste ${ }^{\prime}$, many types of bacteria have survived by receiving resistance genes from soil communities.

Thus, as illustrated in the figure, there exists a general and efficient bacterial mechanism for solving problems and adapting both at the cellular and pluricellular levels. Genes and cells, most of which are unable to survive in isolation,

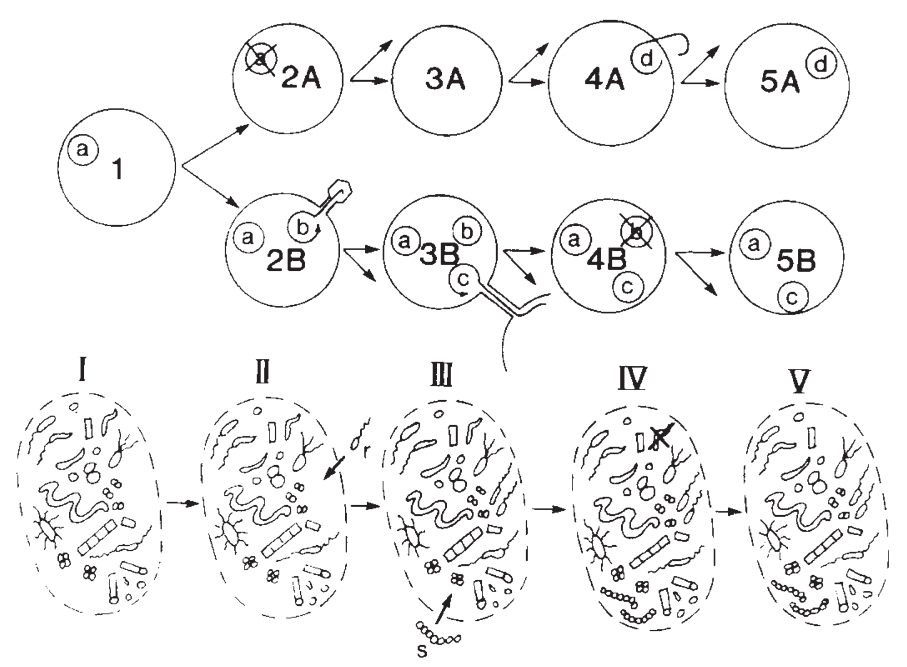

Adaptable chimaeras at cell and community levels following selective pressures: 1 , bacterium with plasmid a; $2 \mathrm{~A}$, a daughter cell loses plasmid; $3 \mathrm{~A}$, a bacterium without plasmids or prophages (rare); 4A, small replicon d arrives by transfection; $5 \mathrm{~A}$, replicon is integrated. $2 \mathrm{~B}$, other daughter cell receives prophage b; $3 \mathrm{~B}$, one of its daughter cells receives a conjugation plasmid, c; 4B, prophage $\mathrm{b}$ is lost again; $5 \mathrm{~B}$, the result. I, A local community of metabolically different bacterial strains represented by different morphologies; II, strain r joins the community; III, r is integrated and multiples, strain s joins; IV and V, strain t disappears as s is integrated.

behave like easily associating basic components. Their potential is realized when they are combined into the best possible dynamic adaptable chimaeras. Only these are effective in nature and in step with permanent competition among bacteria. This general mechanism is suggestive of a computerized communication network ${ }^{2}$. The information bank consists of all the bacterial genes. Selection proceeds through competition. Amplification results from a higher multiplication of the right combination. There are infinite natural circuits to facilitate the flow of genes or of cells. The chief role played by the bacterial world is based on such mechanisms. This simple generalized way of life enables bacteria to avoid extinction.

There is no close equivalence between the organization of eukaryotes and prokaryotes. The term chimaera, as used here, might be replaced by symbiosis ${ }^{5}$, or metabolic differentiation ${ }^{2}$. The bacterial world seems to have evolved as a single loose and partially unified entity in which no gene or strain is entirely foreign. Therefore, there are no real bacterial species. The evolution of bacterial cells and small replicons available for the formation of efficient adaptable chimaeras took place for the possible benefit of the entire planetary bacterial world.

SORIN SONEA

\section{Department of Microbiology and} Immunology,

Université de Montréal, Montréal, Canada H3C $3 J 7$

1. Bull, A.T. \& Slater, J.H. Microbial Interaction and Communities (Academic, London, 1982).
Sonea, S. \& Panisset, M. A New Bacteriology (Jones and Bartlett, Boston, 1983)

3. Zillig, W. et al. Nature 313, 789-791 (1985).

4. Zillig, W. et al. Syst. appl. Microbiol. 7, 58-66 (1986). 5. Sonea, S. Ann. N.Y. Acad. Sci. 53, 251-260 (1987).

\section{Supernova $\gamma$-rays}

SIR-A recent letter (Berezinsky, V.S. \& Ginzburg, V.L. Nature 329, 807-809; 1987) about the possibility of observing very-high-energy (VHE) $\gamma$-rays from the supernova SN1987A from the Southern Hemisphere pleaded for the rapid relocation of ground-based VHE $\gamma$-ray telescopes, and a news item (Nature 330, 410; 1987) reported that two universities in Australia and New Zealand are racing to complete the construction of two such telescopes.

For the record, the University of Durham has been operating a large VHE $\gamma$-ray telescope in Narrabri, New South Wales (NSW), for more than a year. This telescope, on the site of the Sydney University cosmic-ray station at Narrabri, NSW, is part of a project, supported by the Science and Engineering Research Council, for $\gamma$-ray astronomy in the Southern and Northern Hemispheres and costing more than $£ 500,000$. The telescope is similar to, but about 50 per cent larger than, that being constructed by the University of Adelaide and comprises 141 mirrors, each of diameter $60 \mathrm{~cm}$, in three groups. The light detectors at the prime foci are the most comprehensive yet used in VHE $\gamma$-ray astronomy and incorporate 21 photomultipliers giving new background control data particularly important for the supernova observations.

The Narrabri telescope has been operating on a planned programme of 